\title{
Quantitative Nondestructive Testing of Wire Rope Using Image Super-Resolution Method and AdaBoost Classifier
}

\author{
Jigang Li $\mathbb{D}^{1,2}$ and Juwei Zhang $\mathbb{D}^{1,2}$ \\ ${ }^{1}$ College of Electrical Engineering, Henan University of Science and Technology, Luoyang 471023, China \\ ${ }^{2}$ Power Electronics Device and System Engineering Laboratory of Henan, Henan University of Science and Technology, \\ Luoyang 471023, China
}

Correspondence should be addressed to Juwei Zhang; juweizhang@163.com

Received 17 June 2019; Revised 6 July 2019; Accepted 25 July 2019; Published 4 August 2019

Academic Editor: Angelo Marcelo Tusset

Copyright (C) 2019 Jigang Li and Juwei Zhang. This is an open access article distributed under the Creative Commons Attribution License, which permits unrestricted use, distribution, and reproduction in any medium, provided the original work is properly cited.

\begin{abstract}
Magnetic flux leakage (MFL) detection is commonly employed to detect wire rope defects. However, nondestructive testing (NDT) of a wire rope still has problems. A wire rope nondestructive testing device based on a double detection board is designed to solve the problems of large volume, complex operations, and limited circumferential resolution due to sensor size in traditional devices. The device adopts two magnetic sensor arrays to form the double detection board and collects the MFL data of the magnetized wire rope. These sensors on the double detection board are staggered and evenly arranged on the circumference of the wire rope. A super-resolution algorithm based on interpolation uses non-subsampled shearlet transform (NSST) combining principal component analysis (PCA) and Gaussian fuzzy logic (GFL) and fuses the data of double detection board to improve the resolution and quality of defect images. Image quality measurement and comparison experiments are designed to verify that defect images are effectively enhanced. An AdaBoost classifier is designed to classify defects by texture features and invariant moments of defect images. The experimental results show that the detection device not only improves the circumferential resolution, but also the operation is simple; the resolution and quality of the defect images are improved by the proposed super-resolution algorithm, and defects are identified by using the AdaBoost classifier.
\end{abstract}

\section{Introduction}

Wire ropes are widely used in industrial applications such as coal, mining, and other industrial applications because of their advantages: good flexibility, high strength, and strong bearing capacity. The safe operation of wire rope affects the safety of industrial production and personnel. Detecting the damage of wire ropes has important social and economic benefits. The nondestructive testing of a wire rope includes electromagnetic detection, ultrasonic detection, optical detection, X-ray detection, and acoustic emission detection methods. The electromagnetic detection method has widely been used and promoted in current research and application. It includes magnetic flux leakage (MFL) detection, eddy current detection, magnetic memory detection, and magnetic particle detection. MFL detection is the most commonly used method due to its simple structure and strong applicability. The principle of MFL detection is based on the distribution of leakage magnetic field generated by the magnetized wire rope. The damage of the wire rope will affect the magnetic flux leakage distribution on the surface, and the damage detection can be realized by measuring the leakage magnetic field [1].

In the MFL detection method, the magnetization method of the wire rope includes coil magnetization $[2,3]$ and permanent magnet magnetization $[4,5]$. Sun et al. [2] proposed an opening electric magnetizer based on the magnetic control for a C-like electric loop-coil. Its magnetization effect was confirmed by simulations and experiments with the designed 3-D models and prototypes. Wu et al. [3] combined Helmholtz-like coils and a custom-made magnetic shield to design the electromagnet magnetizer. Its structural parameters were optimized by the orthogonal test method. Coil magnetization method requires current flow 
into multiple coils to realize magnetization, which will lead to problems of coil heating and complicated operation. Permanent magnet magnetization is easy to operate. And permanent magnets are usually uniformly arranged around the circumference of the wire rope for magnetization. Sun et al. [4] proposed an open permanent magnet magnetization method, which solved the problem of strong magnetic force and large weight in the yoke magnetic method. Yu et al. [5] considered the effects of excitation system on the performance of leakage magnetic nondestructive testing and optimized the dimensions of the yoke. Wang et al. [6] combined the structural models for dynamic magnetic field balancing and magnetic focusing, which effectively reduced the interference produced by the interactions between the environmental magnetic fields and the wire rope strand. Pan et al. [7] proposed a simple and portable magnetic detector device, which was very light and easy to design and manufacture.

The leakage magnetic field on the surface of the wire rope is usually collected by magnetic sensors, which includes Hall sensor [8], coil sensor [9], fluxgate sensor [10], giant magnetoresistance (GMR) sensor [11], tunnel magnetoresistance (TMR) sensor [12]. The circumferential resolution and sensitivity of magnetic sensors array are important for the detection of wire rope damage. The circumferential resolution of magnetic sensor array affects the location of wire rope damage. Kim and Park [8] designed a hall sensor array to detect wire rope damage; the MFL signal at the defect was enveloped and compared with the threshold to quantify the defect. However, the low circumferential resolution due to Hall sensor size made it difficult to accurately locate the circumferential position of the damage. And the sensitivity of the magnetic sensor also affects the effectiveness of detecting damages. Yan et al. [9] designed a kind of iron core as coil winding skeleton, and their design improved the signal-to-noise ratio (SNR) of magnetic flux leakage signal and simplified coil sensor. Lei et al. [11] proposed a detection device based on high-sensitivity GMR sensors, which solved the problem of low SNR caused by the small diameter and large lift off. Liu et al. [12] designed a circular TMR-based MFL sensor for slight wire rope flaw detection and detected accurately the axial and circumferential positions of these broken wire flaws.

After the magnetic sensors obtain the leakage magnetic field on the surface of the wire rope, signal processing and image processing techniques are used to achieve qualitative and quantitative detection of defects. The MFL signal of the wire rope contains various background noises. The signal processing methods are used to filter out these noises, which can achieve the qualitative analysis of defects. Zhang et al. [13] designed a wavelet filtering algorithm combining the Hilbert-Huang transformation with compression sensing, which effectively suppress the system noise. Wang [14] used the wavelet method to analyze the magnetic flux leakage signal at the defect and verified the wavelet base $\mathrm{db} 4$ with the best noise reduction effect. Liu et al. [15] proposed a wire rope detection signal processing method combining notch filter and wavelet denoising, which could effectively distinguish wire rope defect signal and strand signal, with high detection accuracy, even for the inner defect. Image processing technology can visualize the magnetic flux leakage signal of defects and improve the quality and resolution of defect images, which is important for the quantitative analysis of defects. Zhao et al. [16] used circumferential interpolation to improve the circumferential resolution of the original MFL images and achieved good detection results. Zhang et al. [17] proposed wavelet super-resolution technology to improve the resolution of the defect image, making the edge information of the defect more obvious and easier to identify. Tan and Zhang [18] proposed a superresolution reconstruction method based on Tikhonov regularization to enhance defect image and verified that image property was the best when super-resolution result was triple. However, this method did not apply the exact image quality measurement experiments to verify the effect of defect image enhancement. In addition to these image processing techniques to improve resolution, some image fusion methods can also be used to enhance the MFL image of wire rope defects. In the field of image fusion, traditional multiscale image representation methods have pyramid, wavelet, curvelet, and shearlet. Among them, discrete wavelet transform is a common method, but it has some disadvantages such as not shift invariance, poor directionality, and not a time-invariant transform [19]. Compared to the wavelet transform, contourlet transform has good multiresolution and directionality, but it lacks shift invariance. Non-subsampled contourlet transform is proposed to solve this problem, which adds shift invariance to the contourlet transform, and it can better express the edge and texture information of the image [20]. However, it is not efficient and takes a long time. To solve this problem, NSST is proposed. It not only has short running time and can meet the requirements of real-time [21], but also can extract more detailed features of the target image in different directions.

In order to solve the problems of large volume, complex operations, and limited circumferential resolution due to sensor size in traditional devices, a MFL detection device based on the double detection board is designed. The double detection board consists of magnetic sensors, and these sensors are staggered and evenly arranged on the circumference of the wire rope. The wire rope is magnetized by permanent magnets, and the double detection board collects the surface MFL data of the wire rope. A super-resolution algorithm based on interpolation is applied to double the resolution of defect images. NSST is applied to super-resolution images, which are decomposed into high-frequency coefficients and low-frequency coefficients. For high-frequency coefficients, PCA is implemented as a fusion rule. For low-frequency coefficients, GFL is implemented as a fusion rule. After fusing these coefficients, inverse NSST is applied to reconstruct the fused image. The proposed algorithm fuses the data of double detection board to improve the quality of defect images. Various image quality measurement and comparison experiments are performed, and the results show that spatial resolution of defect images is enhanced effectively and high-quality images are obtained. Image descriptions of texture features and invariant moments are extracted as the feature vector of the defect, which 
are the input of the AdaBoost classifier and are used to identify the defects.

\section{Experimental Design}

2.1. Experimental Platform. The principle of the whole experiment is based on the unsaturated magnetic excitation detection method. Under the excitation state, magnetic field lines generated by the permanent magnet pass through the air inside the excitation rope to form magnetic loops, and there are weak MFL signals at defect as shown in Figure 1(a). Defect information is analyzed by these weak MFL signals. Because the unsaturated magnetic excitation detection method can obtain smoother defect flux leakage signals than the remanence detection method [18], it is applied in this paper. More details about unsaturated magnetic excitation methods can also be found in [18].

The principle of the double detection board is to obtain more circumferential magnetic flux leakage information through the two detection boards. A single detection board limits the number of sensors that can be accommodated due to the sensor size, which also limits the collection of circumferential MFL information of the wire rope. Two detection boards are interlaced around the wire rope, which can collect more circumferential MFL information than one detection board. Double detection board combines with unsaturated excitation detection method constituting the whole experimental platform.

The whole experimental platform is designed as shown in Figure 1(b). The system mainly includes unsaturated magnetic excitation module, double detection board, encoder, data storage, and control system.

The unsaturated magnetic excitation module contains 12 permanent magnets. Permanent magnet material is $\mathrm{NdFeB}$, remanence strength is 1.18 Tesla, and magnetization distance is $1.5 \mathrm{~cm}$. Multiple elongated permanent magnets are evenly distributed around the circumference of the wire rope, and the wire rope is in the excited state. The double detection board consists of two sensor arrays as show in Figure 2. And each sensor array is made of 18 giant magnetoresistance (GMR) sensors. The number of sensors is determined by the lift off distance and sensor size [18]. In order to ensure that two sensor arrays do not repeatedly collect the MFL data on the surface of the wire rope, the circumferential angle of the sensor is $\theta\left(\theta=10^{\circ}\right)$, and the axial distance between the two sensor arrays is $L$ ( $L$ is set to $1 \mathrm{~cm}$ ) as shown in Figure 2. As shown in Figure 3 , these sensors on the double detection board are staggered and evenly arranged on the circumference of the wire rope. The encoder generates control pulses to guarantee the equal spatial sampling. In addition, an ARM chip is used as the control chip, and secure digital (SD) memory card is used for the data storage.

2.2. Experimental Flow. The acquisition processes of the double detection board are as follows: the wire rope is magnetized by the excitation module, the double detection board and the encoder are connected with the control system, and entire acquisition system is loaded onto the magnetized wire rope. When the encoder moves along the wire rope axis, it sends out pulse signal. According to the encoder pulse signal, the double detection board is controlled to collect MFL data on the wire rope surface. There is an interval between the double detection board, which will cause the collected data to be of the same length, but the collected data on the surface of the wire rope are not completely coincident. Therefore, the MFL data of the double detection board cannot be simply superimposed together for subsequent processing. In order to improve the resolution of defect images by fusing the data of double detection board, data of the double detection board are divided into the first board data and the second board data. The double detection board is grouped for data processing and image processing to realize the quantitative identification of wire rope defects, and the processing steps are shown in Figure 4.

\section{Data Processing}

In the experiment, the diameter of steel wire rope is $28 \mathrm{~mm}$ and the structure is $6 \times 37$, and a total of 222 steel wires were used. Broken wires are the main form of wire rope breakage, and it is more difficult to identify the broken wires with small spacing, so it is more meaningful to identify the broken wires with small spacing. Artificial defect types included one discontinuity to five and seven broken wires as shown in Figure 5. And each defect is destroyed as small gap (about $0.2 \mathrm{~cm}$ ).

MFL data on the surface of wire rope collected by the experiment are shown as Figure 6. The original MFL data contain a lot of system noise. These noises include highfrequency magnetic flux leakage noise caused by uneven excitation between sensor channels, baseline drift caused by change of lift off, namely, low-frequency noise, and wave noise caused by spiral structure of the wire rope. These noises will affect the subsequent defect location and quantitative identification results as well as the repeatability of the results. In order to suppress these noises, an effective noise reduction algorithm is needed. Wavelet analysis has widely been used in digital signal processing. In this section, the data processing mainly includes the wavelet soft threshold denoising algorithm for the wire rope MFL signal processing.

Wavelet analysis [14, 15] has been well applied in wire rope MFL signal processing and proved to be an effective signal processing method. In this paper, wavelet analysis is used to decompose the MFL signal of the wire rope, and the high- and low-frequency coefficients of the signal are obtained, in which the high-frequency coefficient contains highfrequency magnetic flux leakage noise and wave noise and the low-frequency coefficient is the baseline. The high-frequency coefficient is treated by soft threshold, and the low-frequency coefficient is cleared. The proposed algorithm is as follows.

(1) Select the $n$-th board MFL data $(n=1,2)$.

(2) Using db4 wavelet to decompose the data $d(i)$ of a sensor channel with 7 level $(i=1-18)$, the data are shown in Figure 7, and wavelet decomposition is as follows: 


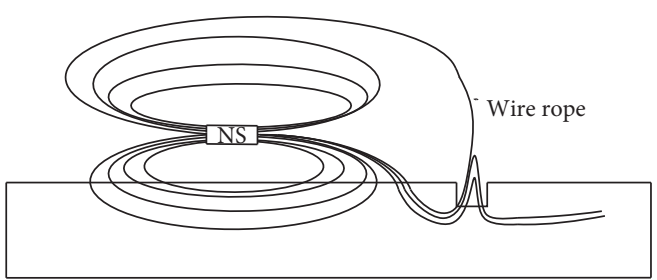

(a)

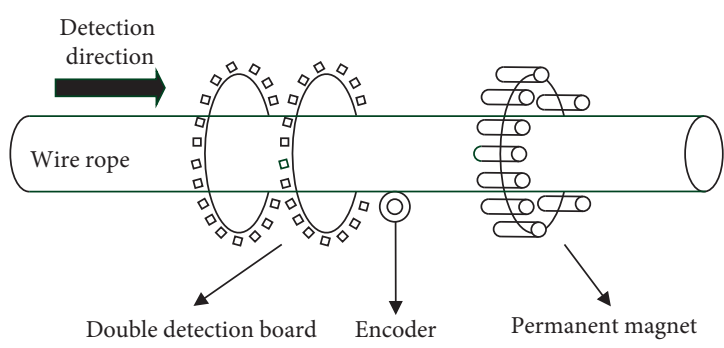

(b)

FIgURE 1: (a) The principle of the unsaturated magnetic excitation detection method. (b) Data acquisition platform schematic.

$$
\begin{array}{r}
\left\{\begin{array}{l}
\bar{x}_{A_{j+1}}=\sum h_{\mathrm{o}}(n-2 k) \bar{x}_{A_{j}}, \\
\bar{x}_{D_{j+1}}=\sum h_{\mathrm{l}}(n-2 k) \bar{x}_{A_{j}},
\end{array}\right. \\
\hat{x}_{A_{j}}=\sum h_{\mathrm{o}}(k-2 n) \bar{x}_{A_{j+1}}+h_{\mathrm{l}}(k-2 n) \bar{x}_{D_{j+1}},
\end{array}
$$

where $h_{\mathrm{o}}$ is the low-pass filter, $h_{1}$ is the high-pass filter, and $\bar{h}_{\mathrm{o}}(k)=h_{1}(-k) ; \bar{x}_{A_{j+1}}$ is the $j$-th low-frequency coefficient; $\bar{x}_{D_{j+1}}$ is the $j$-th high-frequency coefficient; and $\widehat{x}_{A_{j}}$ is the reconstructed signal.

(3) The low-frequency wavelet coefficient is cleared.

(4) The high-frequency coefficients of each decomposition layer are processed by wavelet soft threshold, the universal threshold is "minimaxi."

(5) The processing wavelet coefficients are reconstructed by using the reconstruction equation (2), with which the denoising data are obtained as shown in Figure 8.

\section{Image Processing}

The procedure of image processing in this section mainly includes the image preprocessing and image enhancement. The MFL data collected by the double detection board are grouped into the first board data and the second board data. The first board data are processed by data processing and image preprocessing to obtain a defect image. The second board data are also processed by the same to obtain another defect image. The two defect images show the same defect, but they represent different information of the same defect. The quality of these two images is not good enough and the resolution is not enough. These two images are processed by image enhancement, which fuses different information to obtain a defect image with higher resolution and better quality.

4.1. Image Preprocessing. The image preprocessing mainly includes the defect location and segmentation, the gray-scale normalization, and circumferential interpolation. The location and segmentation of defects were performed by the modulus maxima method [17]. The modulus maxima method is used to obtain the axial position information of the defect regions. The defect images are segmented according to the axial information of the defects region. The gray-scale normalization can transform defect images into defect gray

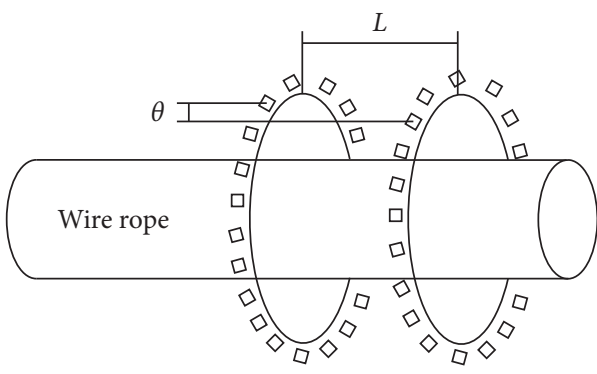

Figure 2: Double detection board schematic.

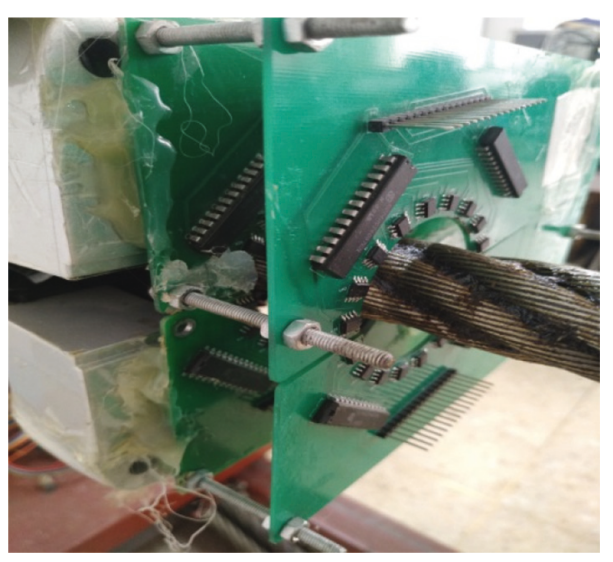

Figure 3: Double detection board.

images. Defect images are normalized to $[0,255]$ by the maxmin normalization method, which converts MFL data into defect image. Each sensor array in the double detection board has 18 channels. Therefore, the circumferential resolution of defect image is only 18, which is far below the axial resolution. In order to make the defect image more intuitive, cubic spline interpolation method is used to improve the circumferential resolution from 18 to 300 .

After the above image preprocessing, two images of the same defect are finally obtained. The specific process of image preprocessing is as follows:

(1) Select the $n$-th board data after noise reduction $(n=1,2)$ as shown in Figure 9

(2) Set the threshold to obtain the channel where the defect is located, sum up all defect channels, and 


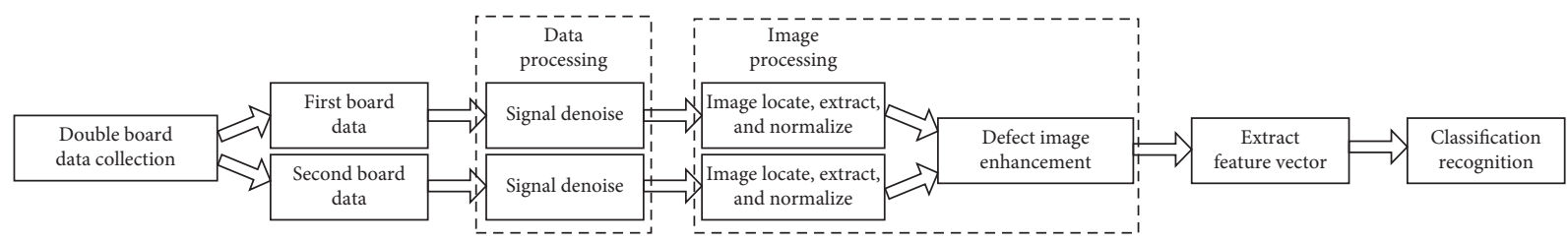

Figure 4: Defect recognition flow chart.

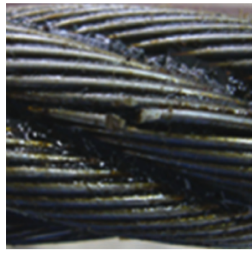

(a)

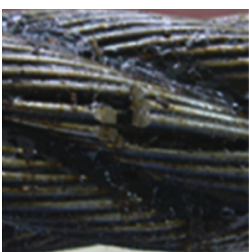

(c)

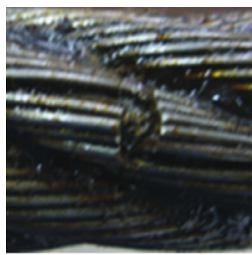

(e)

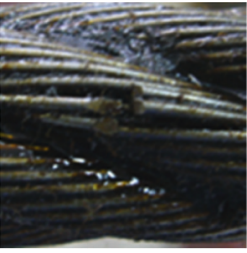

(b)

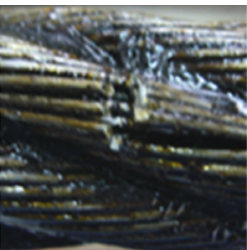

(d)

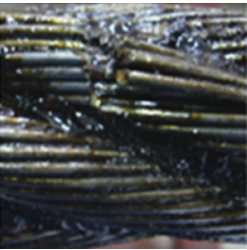

(f)

Figure 5: Images of broken wires. (a) One broken wire. (b) Two broken wires. (c) Three broken wires. (d) Four broken wires. (e) Five broken wires. (f) Seven broken wires.

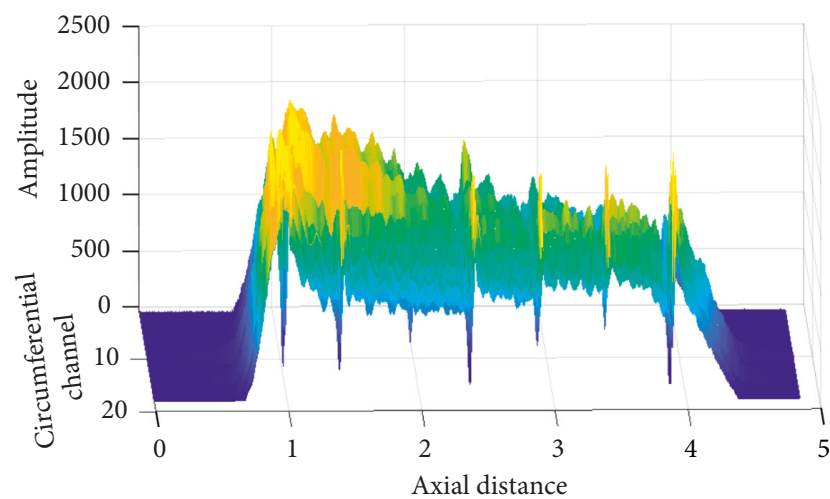

(a)

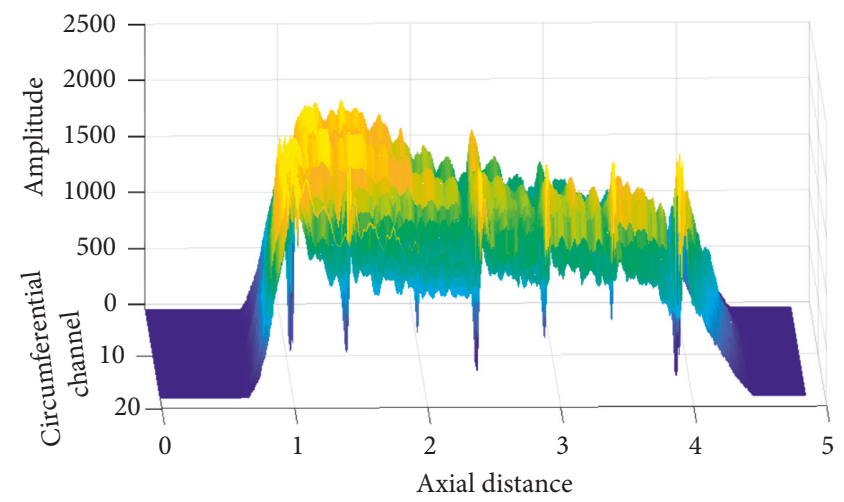

(b)

Figure 6: The original MFL data. (a) The first board. (b) The second board.

take the absolute value, where the position with the maximum value is the axial coordinates of the defect

(3) The $18 \times 300$ defect images is segmented according to the axial coordinates of the defect, which are centered on these center points, and the defect regions are shifted to the center along the circumferential direction of the defect images

(4) The defect images are normalized to $[0,255]$ to obtain the defect gray images 


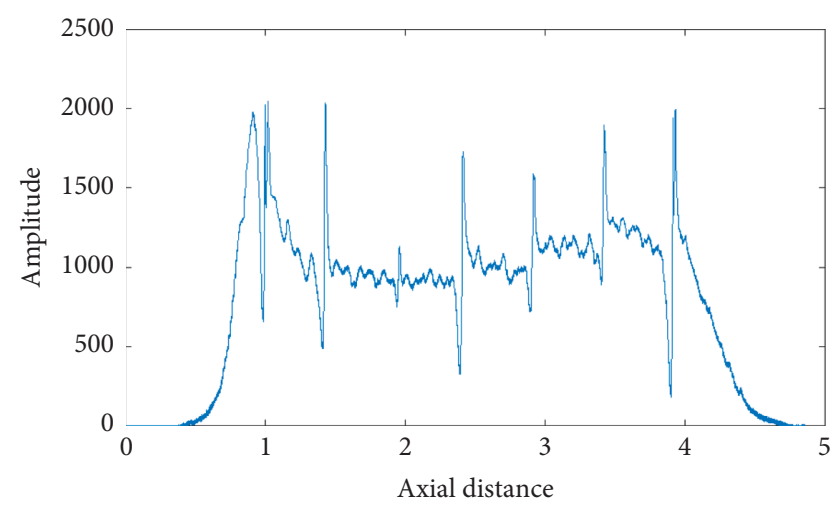

FIgURE 7: The original MFL data of single channel.

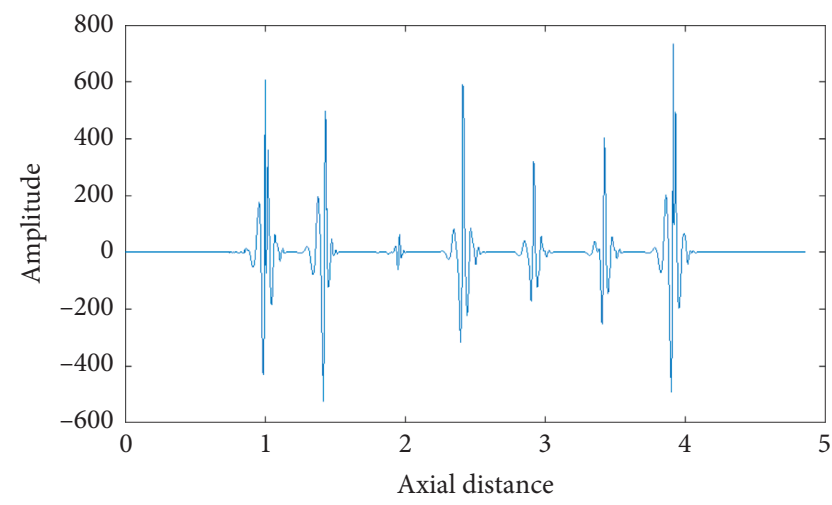

Figure 8: The denoising data of single channel.

(5) Cubic spline interpolation is used to improve the circumferential resolution of defect gray images from 18 to 300

(6) The $300 \times 300$ defect gray images of the same defect are finally obtained as shown in Figure 10

4.2. Image Enhancement. In this section, image enhancement mainly uses the super-resolution algorithm-based interpolation to fuse two images of the same defect. The algorithm not only improves the resolution of the defect image but also improves the quality. The flow chart of proposed algorithm is shown in Figure 11. High-resolution and highquality images can provide more details about defects. More details can improve the distance between the characteristics of the defect images, making the defect image easier to classify improving the accuracy of quantitative recognition.

The super-resolution method can transform the lowresolution image into the high-resolution image and has widely been used in image enhancement. Image interpolation is widely used in image super-resolution due to its simplicity and speed. Traditional interpolation methods include nearest neighbor interpolation, bicubic interpolation, and bilinear interpolation. Among them, bicubic interpolation is the best because bicubic interpolation can produce smoother edges than the others [22]. In this paper, the super-resolution method based on bicubic interpolation is used to improve the resolution of two images of the same defect. The resolution of each image is doubled, which can continue to increase, but this situation will increase the computational cost.

After obtaining two high-resolution images at the same defect, NSST is used to fuse two high-resolution images at the same defect. And NSST theory is explained in Section 4.2.1. NSST is applied to two high-resolution images at the same defect separately. Each image is decomposed into its corresponding high-frequency and low-frequency coefficients. Then, the fusion of the high-frequency coefficients and the low-frequency coefficients of the two pictures are, respectively, performed by different fusion rules. The high-frequency coefficient represents the details of image, PCA is used as fusion rule. And the rule is explained in Section 4.2.2. The low-frequency coefficients represent the contour of the image, and GFL is used as fusion rule and is explained in Section 4.2.3. After fusing the coefficients, inverse NSST is applied to reconstruct the fused image. Because of the super-resolution method, the size of the fused image is bigger than the original defect image. To measure quality objectively, the fused image is resized to the size of original source image using interpolation-based resizing method. And finally, the fused image with the original size is created and is ready for image quality measurement.

4.2.1. Non-Subsampled Shearlet Transform. NSST uses nonsubsampled pyramid filters (NSPFs) to decompose the input image into different scales. If image is decomposed in $L$ level, $L+1$ subbands of the same size as the input image will be obtained, including $L$ high-frequency subbands and one low-frequency subband. For each decomposition level, shiftinvariant shearlet filter banks (SFBs) are used to decompose subbands into different directional subbands. More details about NSST can be found in the literature [23]. Due to the characteristics of NSST such as multiscale, multidirection, and shift invariance, it is selected as the proposed fusion method in this paper.

4.2.2. Principal Component Analysis. PCA can convert a large number of related variables into unrelated variables. That means this method can reduce the redundant data and extract the important parts of images, so it is widely used in the field of image fusion. PCA uses a weighted average of images to fuse these source images, and the weights depends on the eigenvector corresponding to the largest eigenvalue of the covariance matrices of each source image. And PCA is selected as fusion rule of high-frequency coefficients in this paper. More details about PCA can be found in the literature [24]. The PCA algorithm steps are shortly defined as follows:

(1) Let the source images (images to be fused) be arranged in two-column vectors

(2) Subtract the mean of each column from the twocolumn vectors

(3) Calculate covariance matrixes of the two-column vectors

(4) Calculate eigenvectors $V$ of covariance matrixes, and $V$ is diagonal matrix with dimension $2 \times 2$ 


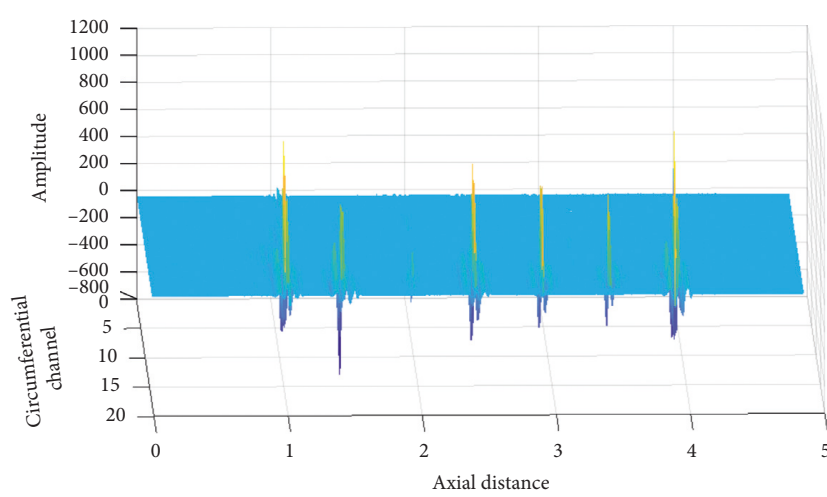

(a)

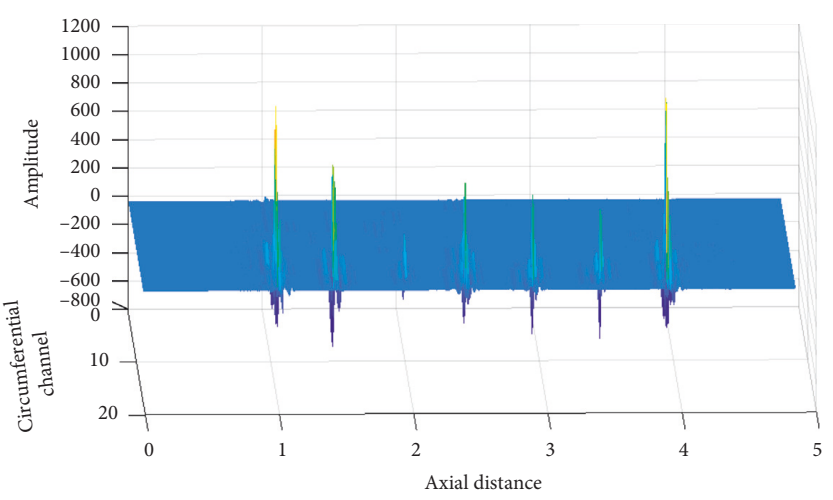

(b)

Figure 9: The original denoising wire rope MFL image. (a) The first board. (b) The second board.

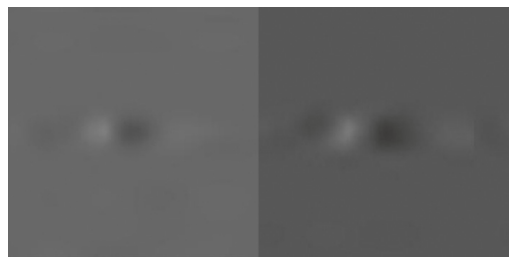

(a)

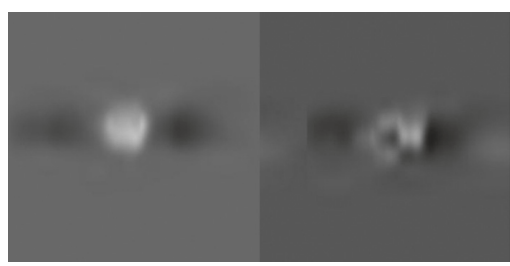

(c)

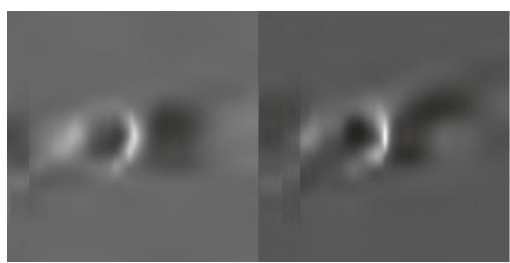

(e)

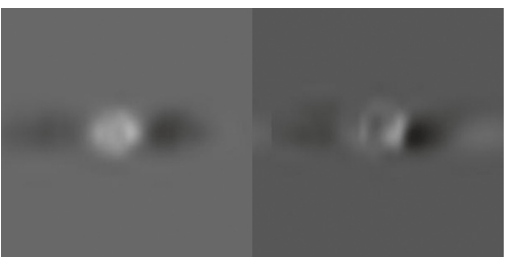

(b)

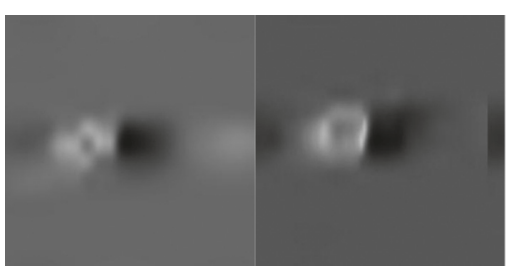

(d)

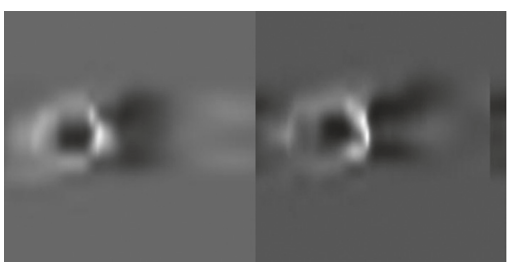

(f)

Figure 10: The two gray-scale images of the same defect. (a) One broken wire. (b) Two broken wires. (c) Three broken wires. (d) Four broken wires. (e) Five broken wires. (f) Seven broken wires.

(5) Consider eigenvalues of $V$ which correspond to $V(1)$ and $V(2)$ to compute $p_{1}$ and $p_{2}$ as

$$
\begin{aligned}
& p_{1}=\frac{V_{1}}{\sum V}, \\
& p_{2}=\frac{V_{2}}{\sum V} .
\end{aligned}
$$

The fusion rule about PCA is as follows:

$$
f_{k}^{d}(i, j)=p_{1} f_{A, k}^{d}(i, j)+p_{2} f_{B, k}^{d}(i, j),
$$

where $A$ and $B$, respectively, denote source images 1 and 2 and $f_{A, k}^{d}$ and $f_{B, k}^{d}$ are the high-frequency subimages of source images 1 and 2, respectively.

4.2.3. Gaussian Fuzzy Logic. GFL has been well applied in image fusion. The low-frequency coefficient of the source image contains the target information and background information of the image. GFL can select the feature target information of the source image and complement the background information of another source image. By using the weighted average method based on GFL to fuse the image 


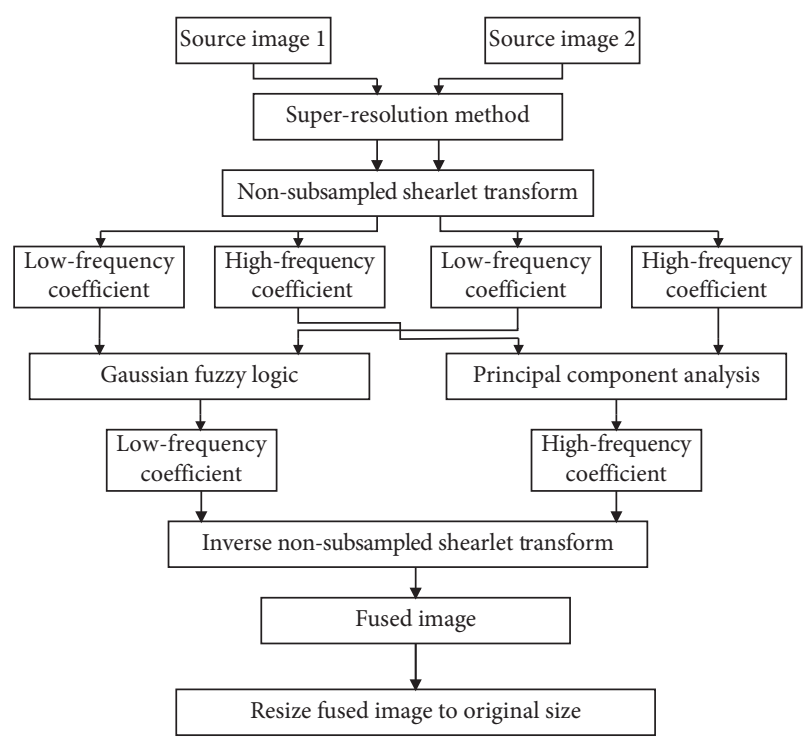

FIgURE 11: Flow chart of the proposed algorithm.

low-frequency coefficients, the main information and contour information of the source image can be retained to the greatest extent. So GFL is selected as fusion rule of low-frequency coefficients in this paper. More details about GFL can be found in the literature [25]. GFL formulas are as follows:

$$
\begin{aligned}
\eta_{0} & =\exp \left(-\frac{\left(f_{A}(i, j)-\mu\right)^{2}}{2(k \sigma)^{2}}\right), \\
\eta_{1} & =1-\eta_{0}, \\
f(i, j) & =\eta_{0} f_{A}(i, j)+\eta_{1} f_{B}(i, j),
\end{aligned}
$$

where $\mu$ and $\sigma$, respectively, are the mean and variance of the source image $1 ; k$ is a constant and is set to 1.5 ; and $f_{A}$ and
$f_{B}$ are the low-frequency subimages of source images 1 and 2 , respectively.

4.3. Image Quality Measurement and Comparison. Since the defect gray image is relatively simple and intuitive, it is not easy to see the quality changes before and after the enhancement of the image quality and resolution. To measure the visual effect of the enhanced image, eight metrics are applied to make a comprehensive evaluation. These evaluation measures include average gradient [25], information entropy [25], standard deviation [25], space infrequency [25], mutual information [26], Petrovics metric [26], signal-to-noise ratio [24], and structural similarity index measure [24] as follows:

4.3.1. Image Quality Measurement. Image quality measurement experiment is performed to prove the effectiveness of the proposed super-resolution algorithm for defect images enhancement. In the experiment, images with improved resolution and two source images were used for quality measurement. Four groups of quality measurement indicators were selected as shown in equations (6)-(10), and eight groups of different images were measured in each group. The experimental results are shown in Tables 1-4, and the average results in these tables are shown in Table 5.

Since the resolution of the image improved by the algorithm is inconsistent with that of the source image, which affects the quality measurement comparison, the image with the improved resolution is adjusted to the size of the source image, and the adjusted image is used for experiments. In these tables, B1 and B2 represent source images 1 and 2, respectively, as shown in Figure 11, and the two source images represent the data collected by double detection boards, respectively. And B3 represents the adjusted image.

(1) Average gradient (AG):

$$
\mathrm{AG}=\frac{1}{m \times n} \sqrt{\frac{\left(\sum_{i-1}^{m} \sum_{j-2}^{n}(F(i, j)-F(i, j-1))\right)^{2}+\left(\sum_{i-2}^{m} \sum_{j-1}^{n}(F(i, j)-F(i-1, j))\right)^{2}}{2}}
$$

where $F$ denotes the final adjusted image whose size is $m \times n$.

(2) Information entropy (IE):

$$
\mathrm{IE}=-\sum_{i=0}^{L} p(i) \log _{2}(p(i))
$$

where $p(i)$ denotes the probability of pixels whose gray value amount to $i$ over the total image pixels.

(3) Standard deviation (SD):

$$
\mathrm{SD}=\sqrt{\frac{1}{m \times n} \sum_{i-1}^{m} \sum_{j-1}^{n}\left[F(i, j)-\frac{1}{m \times n} \sum_{i-i}^{m} \sum_{j-1}^{n} F(i, j)\right]^{2}}
$$

(4) Space infrequency (SF):

$$
\begin{aligned}
\mathrm{SF} & =\sqrt{\mathrm{RF}^{2}+\mathrm{CF}^{2}}, \\
\mathrm{RF} & =\sqrt{\frac{1}{m \times n} \sum_{i=2}^{m} \sum_{j=1}^{n}[F(i, j)-F(i-1, j)]^{2}}, \\
\mathrm{CF} & =\sqrt{\frac{1}{m \times n} \sum_{i=1}^{m} \sum_{j=2}^{n}[F(i, j)-F(i, j-1)]^{2}},
\end{aligned}
$$

where $\mathrm{RF}$ and $\mathrm{CF}$, respectively, denote the row frequency and column frequency. 
TABLE 1: AG.

\begin{tabular}{lccccccccc}
\hline Group & 1 & 2 & 3 & 4 & 5 & 6 & 7 & 8 & Average \\
\hline B1 & 0.0035 & 0.0029 & 0.0024 & 0.0040 & 0.0025 & 0.0071 & 0.0038 & 0.0031 & 0.0037 \\
B2 & 0.0035 & 0.0031 & 0.0024 & 0.0033 & 0.0028 & 0.0055 & 0.0045 & 0.0056 & 0.0038 \\
B3 & 0.0039 & 0.0035 & 0.0031 & 0.0042 & 0.0034 & 0.0075 & 0.0053 & 0.0041 & 0.0044 \\
\hline
\end{tabular}

TABle 2: IE.

\begin{tabular}{lccccccccc}
\hline Group & 1 & 2 & 3 & 4 & 5 & 6 & 7 & 8 & Average \\
\hline B1 & 4.1983 & 3.9262 & 2.5703 & 4.5355 & 4.2720 & 3.8935 & 4.4978 & 3.9865 & 3.9850 \\
B2 & 4.1687 & 3.8971 & 2.3877 & 4.2999 & 4.5391 & 4.2002 & 4.9572 & 3.9661 & 4.0520 \\
B3 & 4.2574 & 3.9736 & 2.4467 & 4.3588 & 4.5574 & 4.1548 & 5.0237 & 4.0170 & 4.0987 \\
\hline
\end{tabular}

TABLE 3: SD.

\begin{tabular}{|c|c|c|c|c|c|c|c|c|c|}
\hline Group & 1 & 2 & 3 & 4 & 5 & 6 & 7 & 8 & Average \\
\hline B1 & 17.9798 & 15.5389 & 12.2226 & 20.0783 & 13.6876 & 18.8431 & 20.0422 & 13.0041 & 16.4246 \\
\hline B2 & 12.7756 & 15.2936 & 10.9179 & 17.2332 & 14.3510 & 21.0038 & 19.1639 & 14.0454 & 15.5980 \\
\hline B3 & 17.8231 & 16.0374 & 12.9759 & 20.5396 & 14.7274 & 20.0267 & 20.8514 & 14.5853 & 17.1959 \\
\hline
\end{tabular}

TABLE 4: SF.

\begin{tabular}{lccccccccc}
\hline Group & 1 & 2 & 3 & 4 & 5 & 6 & 7 & 8 & Average \\
\hline B1 & 1.4750 & 1.2277 & 1.0097 & 1.6777 & 1.0677 & 2.9947 & 1.6255 & 1.3136 & 1.5490 \\
B2 & 1.4920 & 1.3226 & 0.9942 & 1.4112 & 1.1600 & 2.3327 & 1.8937 & 2.3527 & 1.6199 \\
B3 & 1.6405 & 1.4736 & 1.3011 & 1.7523 & 1.4283 & 3.1750 & 2.2749 & 1.7417 & 1.8484 \\
\hline
\end{tabular}

TABLE 5: The average results.

\begin{tabular}{lcccc}
\hline Measure & AG & IE & SD & SF \\
\hline B1 & 0.0037 & 3.9850 & 16.4246 & 1.5490 \\
B2 & 0.0038 & 4.0520 & 15.5980 & 1.6199 \\
B3 & 0.0044 & 4.0987 & 17.1959 & 1.8484 \\
\hline
\end{tabular}

The results from Tables $1-5$, especially Table 5, show that all four quality measures of B3 exceed those of B1 and B2, which means that the size adjusted images are of better quality than the two source images. Experimental results show that the proposed super-resolution algorithm can effectively improve the quality and resolution of defect images.

4.3.2. Image Quality Comparison. Image quality comparison experiment is to prove the feasibility of the proposed super-resolution algorithm for defect image enhancement. The performance of the proposed algorithm is compared with several algorithms: bicubic interpolation (BI), wavelet super-resolution reconstruction (WSR) [17], stationary wavelet transform super-resolution method (SWTSR) [26], and non-subsampled contourlet transform super-resolution reconstruction (NSCTSR) [27].
Two source images were used to test these superresolution algorithms, and their super-resolution renderings are shown in Figure 12. It is not easy to see the difference of image quality from these super-resolution results, which is due to the simple structure and texture of defect gray-scale images. Therefore, four measurement indexes were used to measure these super-resolution result images. The four measurement indicators are as shown in equations (11)-(17). Various super-resolution measurement results are shown in Table 6.

(5) Mutual information (MI):

$$
\begin{gathered}
\mathrm{MI}=\mathrm{MI}_{A, F}+\mathrm{MI}_{B, F}, \\
\mathrm{MI}_{X, F}=\sum_{x, f} p_{X, F}(x, f) \log \frac{p_{X, F}(x, f)}{p_{X}(x) p_{F}(f)},
\end{gathered}
$$

where $A$ and $B$, respectively, denote source images 1 and $2, X$ is $A$ or $B$, and $p_{X, F}$ is the normalized gray 


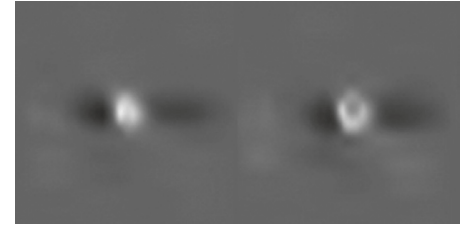

(a)

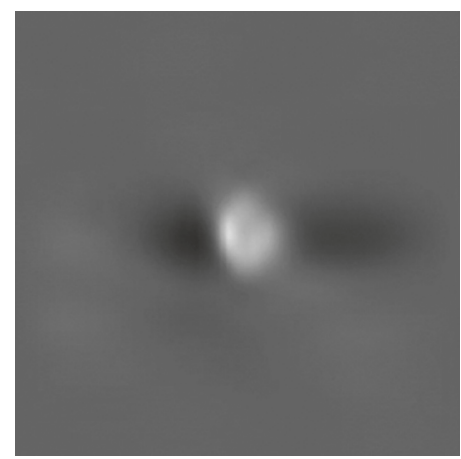

(d)

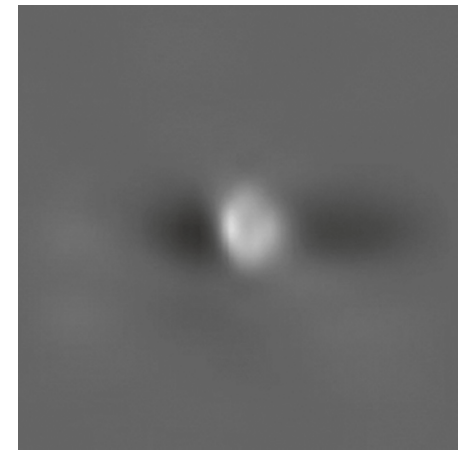

(b)

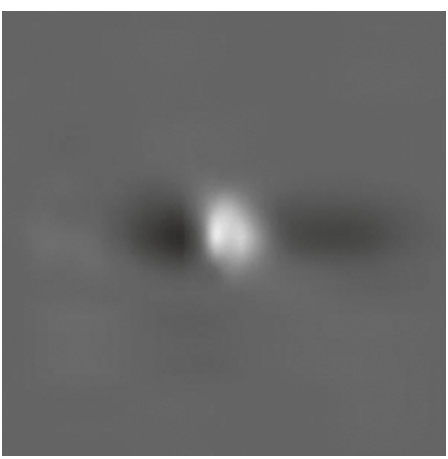

(e)

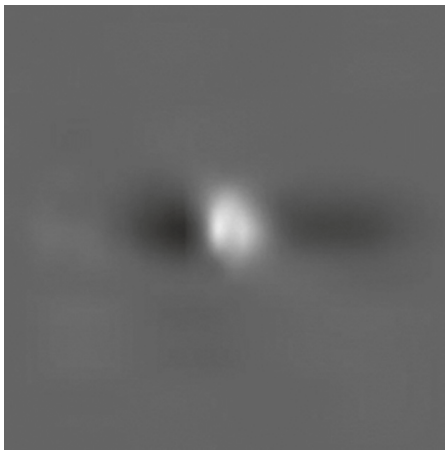

(c)

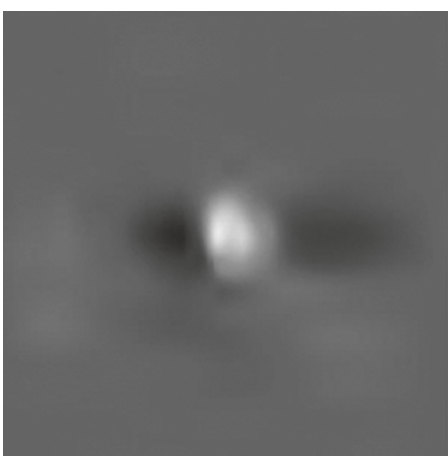

(f)

Figure 12: Various super-resolution results. (a) Two source images. (b) BI. (c) WSR. (d) SWTSR. (e) NSCTSR. (f) Proposed.

TABLE 6: Various super-resolution measurement results.

\begin{tabular}{lcccc}
\hline Measure & MI & $Q^{A B / F}$ & SNR & \\
\hline BI & 4.0838 & 0.6030 & 0.0198 & 0.6163 \\
WSR & 5.1995 & 0.5929 & 0.0011 & 0.6207 \\
SWTSR & 4.1538 & 0.5907 & 0.0064 & 0.5934 \\
NSCTSR & 3.9762 & 0.5573 & 0.0021 & 0.4914 \\
Proposed & 5.3348 & 0.6430 & 0.0403 & 0.6313 \\
\hline
\end{tabular}

histograms of these source images and the fusion image, respectively.

(6) Petrovics metric $\left(Q^{\mathrm{AB} / F}\right)$ :

$$
Q^{A B / F}=\frac{\sum_{i=1}^{N} \sum_{j=1}^{M} Q^{A F}(i, j) w^{A}(i, j)+Q^{B F}(i, j) w^{B}(i, j)}{\sum_{i=1}^{N} \sum_{j=1}^{M}\left(w^{A}(i, j)+w^{B}(i, j)\right)},
$$

where $Q^{A F}$ shows the relation with source image 1 and fused image $F$ looking to edge information. And $w^{A}$ shows the edge strength of source image 1 .

(7) Signal-to-noise ratio (SNR):

$$
\begin{aligned}
& \mathrm{SNR}=\mathrm{SNR}_{A}+\mathrm{SNR}_{B}, \\
& \mathrm{SNR}_{X}=20 \log _{10}\left[\frac{\sum_{i=1}^{M} \sum_{j=1}^{N}\left(f_{X}(i, j)\right)^{2}}{\sum_{i=1}^{M} \sum_{j=1}^{N}\left(f_{X}(i, j)-f(i, j)\right)^{2}}\right] .
\end{aligned}
$$

(8) Structural similarity index measure (SSIM):

$$
\begin{aligned}
\operatorname{SSIM} & =\left(\operatorname{SSIM}_{A, F}+\operatorname{SSIM}_{B, F}\right), \\
\operatorname{SSIM}_{X, F} & =\frac{\left(2 u_{x} u_{f}+C_{1}\right)\left(2 \sigma_{x} \sigma_{f}+C_{2}\right)}{\left(u_{x}^{2}+u_{f}^{2}+C_{1}\right)\left(\sigma_{x}^{2}+\sigma_{f}^{2}+C_{2}\right)} .
\end{aligned}
$$

The results from Table 6 show that all four quality measures of B3 exceed those of B1 and B2, which means that the proposed super-resolution algorithm has a good effect on gray-scale image enhancement of wire rope defects. Experimental results show that the proposed algorithm is feasible to wire rope defect image enhancement.

\section{Quantitative Identification}

Quantitative identification is an important goal of wire rope nondestructive testing. In this part, texture features and 


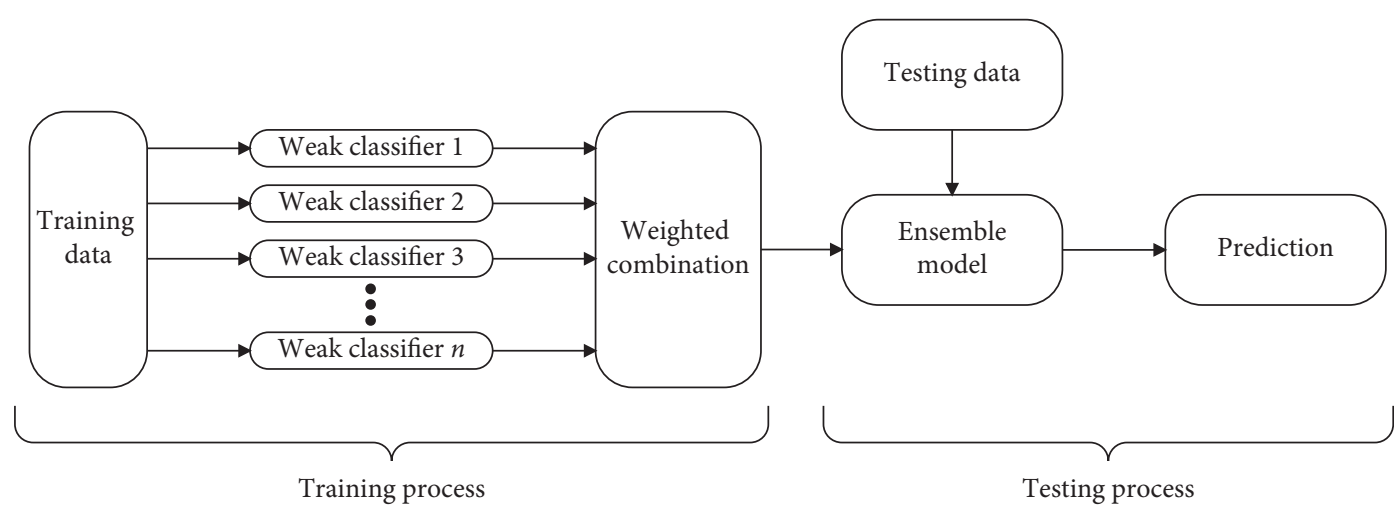

Figure 13: AdaBoost classifier flow chart.

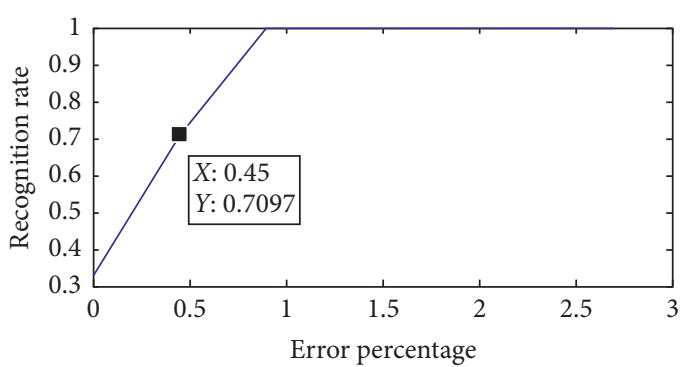

(a)

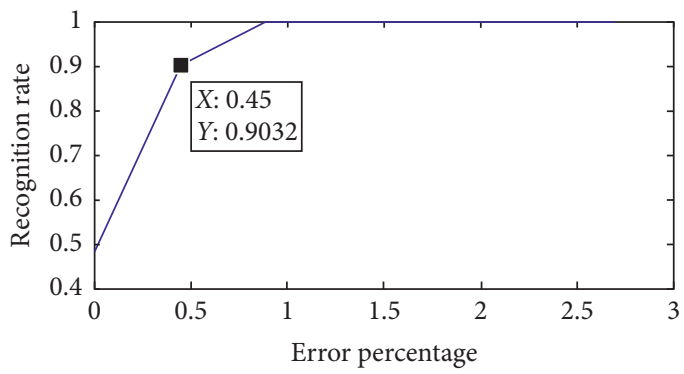

(c)

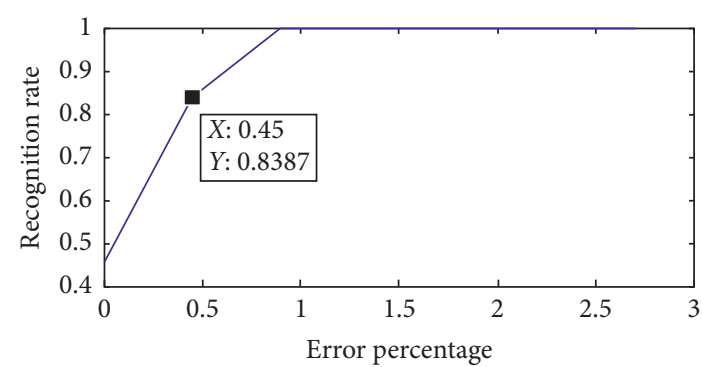

(b)

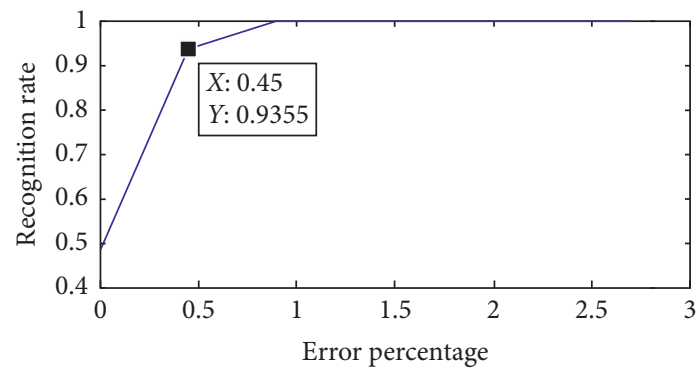

(d)

FIGURE 14: Identification results of broken wires under different decision trees. (a) 10. (b) 20. (c) 40. (d) 60.

seventh-order moment invariant features of defect images are extracted as feature vectors of defect images. These features include standard deviation, smoothness, third-order moment, consistency, and entropy, and the first, third, fifth and seventh moments of seven-order invariant moments. The designed AdaBoost classifier recognizes these defects by the feature vectors of defect images.

5.1. AdaBoost Classifier. The adaptive boosting (AdaBoost) ensemble is to combine a number of weak classifiers to get a strong classifier which has better classification effect. Each weak learner is a simple classifier such as decision tree and neural networks. AdaBoost combines weak learners such as decision trees to make it one of the best classifiers.

AdaBoost is a classifier with high accuracy. It is simple, does not require feature screening, and does not worry about overfitting. The flow chart for AdaBoost is as shown in
Figure 13. In this paper, the weak classifier selects the decision tree. AdaBoost is divided into training process and testing process. In the process of training, the training samples are set as the same initial weights, a weak classifier is trained, and the classification error rate is calculated. Then, the weight values are updated iteratively in each iteration based on the previous classification result, that is, increase the sample weight of wrong classification and reduce the weight of correctly classified samples. If classification error rate is more than or equal to 0.5 , the weight will be reinitialized. Each weak learner has weight which is proportional to the classification error rate. In the process of testing, the test samples are used for these weighted classifiers, and the final classification results are output. More details about AdaBoost can be found in the literature [28].

5.2. Statistics Results. Broken wires are the main damage form of wire rope, and small spacing of broken wires is 
difficult to identify and more meaningful. In the quantitative identification experiment, 125 samples of concentrated broken wires were manufactured manually, with a small spacing of about $0.2 \mathrm{~cm}$, and the types of broken wires included 1 to 5 and 7. The broken wire samples were randomly divided into training samples and test samples. The number of training samples was 94 (about 75\%), and the number of test samples was 31 (about 25\%). In this paper, the number of broken wires identified by AdaBoost classifier is converted into the percentage of broken wires, it represents the percentage of broken wires in the total wires and makes the classification of broken wires more intuitive. As shown in Figure 14, the identification result graph under different number of decision trees has the best recognition effect when the number of decision trees is 60 . When the permissible error of broken wires is $0.45 \%$, which means the permissible error is one wire, the recognition rate of broken wires reaches $93.55 \%$, and the maximum error was not more than $0.9 \%$.

\section{Results and Discussion}

In this paper, a wire rope nondestructive testing device based on the double detection board is designed to collect MFL data of the wire rope. The double detection board can collect more circumferential information of the wire rope surface. A superresolution algorithm combining interpolation and NSST is used to improve the resolution and quality of defect images. The interpolation algorithm uses cubic interpolation to improve the resolution of defect images. NSST decomposes these high-resolution images to get high-frequency and low-frequency images, and GFL fuses low-frequency images and PCA fuses high-frequency images. The super-resolution algorithm fuses the data of the double detection board to produce better quality and higher resolution defect images. Various image quality measurements and comparison experiments are performed to show the effectiveness of the proposed algorithm. Compared with the super-resolution algorithm in literature [17, 26, 27], the proposed algorithm has better image quality improvement effect. After obtaining high-resolution defect images with good quality, the AdaBoost classifier was designed to identify these defect images so as to achieve quantitative recognition of broken wires. When the permissible error of broken wire is $0.45 \%$ (the permissible error is one wire), the highest recognition rate of the broken wire is $93.55 \%$. In comparison with [18], the identification accuracy rate was $91.43 \%$ with the permissible error of one wire. Compared with [13], the accuracy was $93.75 \%$ under a permissible error of two wires. The proposed method outperforms existing methods. Quantitative identification results show that the AdaBoost classifier is feasible and effective for broken wires recognition.

In the experiments, the distance between the double detection board and the excitation source in the data acquisition platform is different, which results in some differences in the signal-to-noise ratio of the MFL data collected by each detection board in the double detection board. Using the same filtering algorithm for the signals collected by the double detection board will lead to the difference of filtering effect, which will affect the image super-resolution enhancement effect. Furthermore, in the image preprocessing part, cubic spline interpolation is used to improve the circumferential resolution of the defect image, while in the image enhancement part, bicubic interpolation is used again to improve the resolution of the image, and so repeated interpolation will affect the defect image quality. Therefore, future research will focus on the optimization of the filtering algorithm and image super-resolution algorithm.

\section{Conclusions}

In this paper, the three research works have been performed. Firstly, a wire rope nondestructive testing device based on the double detection board to address the disadvantages of the traditional detection device. Compared with traditional MFL traditional detection device, the proposed device has small volume, simple operations, and high circumferential resolution. Secondly, a super-resolution algorithm combining interpolation and NSST is used to fuse the MFL data of double detection board to improve the quality of defect images. Various image quality measurements and comparison experiments are performed to show the effectiveness of the proposed algorithm. Finally, the AdaBoost classifier is designed to identify the broken wires quantitatively. The results of quantitative experiment show a good recognition effect of broken wires. In the future, the filtering algorithm and image super-resolution algorithm will continue to be optimized, and more types of damage will be studied.

\section{Data Availability}

The data used to support the findings of this study are available from the corresponding author upon request.

\section{Conflicts of Interest}

The authors declare that there are no conflicts of interest regarding the publication of this paper.

\section{Acknowledgments}

This work was partially supported by the National Natural Science Foundation of China (grant nos. 61040010, 61172014, and U1504617), the Key Technologies R\&D Program of Henan Province (grant no. 152102210284), the Science and Technology Program of Henan Education Department (grant no. 17A510009), and the Science and Technology Open Cooperation Program of Henan Province (grant no. 182106000026).

\section{References}

[1] J. Tian, J. Zhou, H. Wang, and G. Meng, "Literature review of research on the technology of wire rope nondestructive inspection in China and abroad," MATEC Web of Conferences, vol. 22, article 03025, 2015.

[2] Y. Sun, J. Wu, B. Feng, and Y. Kang, "An opening electricMFL detector for the NDT of in-service mine hoist wire," IEEE Sensors Journal, vol. 14, no. 6, pp. 2042-2047, 2014.

[3] B. Wu, Y. J. Wang, X. C. Liu, and C. F. He, "A novel TMRbased MFL sensor for steel wire rope inspection using the 
orthogonal test method," Smart Materials and Structures, vol. 24, no. 7, article 075007, 2015.

[4] Y. Sun, S. Liu, and L. He, "A new detection sensor for wire rope based on open magnetization method," Materials Evaluation, vol. 75, no. 4, pp. 501-509, 2017.

[5] C. Yu, J. Jiao, G. Li, X. Liu, C. He, and B. Wu, "Effects of excitation system on the performance of magnetic-fluxleakage-type non-destructive testing," Sensors \& Actuators A Physical, vol. 268, pp. 201-212, 2017.

[6] H. Wang, J. Tian, and G. Meng, "A sensor model for defect detection in mine hoisting wire ropes based on magnetic focusing," Insight-Non-Destructive Testing and Condition Monitoring, vol. 59, no. 3, pp. 143-148, 2017.

[7] S. Pan, D. Zhang, and E. Zhang, "Nondestructive testing for shallow defect of ferromagnetic objects based on magnetic probe structure," IEEE Transactions on Magnetics, vol. 54, no. 11, pp. 1-6, 2018.

[8] J.-W. Kim and S. Park, "Magnetic flux leakage-based local damage detection and quantification for steel wire rope nondestructive evaluation," Journal of Intelligent Material Systems and Structures, vol. 29, no. 17, pp. 3396-3410, 2018.

[9] X. Yan, D. Zhang, and F. Zhao, "Improve the signal to noise ratio and installation convenience of the inductive coil for wire rope nondestructive testing," NDT \& E International, vol. 92, pp. 221-227, 2017.

[10] J. Li, Y. Wang, X. Zhang, C. Ji, and J. Shi, "Sensitivity and resolution enhancement of coupled-core fluxgate magnetometer by negative feedback," IEEE Transactions on Instrumentation and Measurement, vol. 68, no. 2, pp. 623-631, 2019.

[11] H. M. Lei, R. H Liang, W. Tao, Y.-M. Mao, and H. Zhao, "Broken wires inspection for coated steel belts in elevator system using MFL method," in Proceedings of the 2014 IEEE Far East Forum on Nondestructive Evaluation/Testing, pp. 252-254, Chengdu, China, October 2014.

[12] X. Liu, Y. Wang, B. Wu, G. Zhen, and H. Cunfu, "Design of tunnel magnetoresistive-based circular MFL sensor array for the detection of flaws in steel wire rope," Journal of Sensors, vol. 2016, Article ID 6198065, 8 pages, 2016.

[13] J. Zhang, X. Tan, and P. Zheng, "Non-destructive detection of wire rope discontinuities from residual magnetic field images using the Hilbert-Huang transform and compressed sensing," Sensors, vol. 17, no. 3, p. 608, 2017.

[14] Y. F. Wang, "Research on application of wavelet denoising into broken wire damage detection of mine steel wire rope," in Proceedings of the 35th Chinese Control Conference (CCC), pp. 6644-6648, IEEE, Chengdu, China, August 2016.

[15] S. Liu, Y. Sun, W. Ma et al., "A new signal processing method based on notch filtering and wavelet denoising in wire rope inspection," Journal of Nondestructive Evaluation, vol. 38, no. 2, p. 39, 2019.

[16] M. Zhao, D. L. Zhang, and Z. H. Zhou, "The research on quantitative inspection technology to wire rope defect based on hall sensor array," Nondestructive Testing, vol. 34, no. 11, pp. 57-60, 2012.

[17] J. Zhang, P. Zheng, and X. Tan, "Recognition of broken wire rope based on remanence using EEMD and wavelet methods," Sensors, vol. 18, no. 4, p. 1110, 2018.

[18] X. Tan and J. Zhang, "Evaluation of composite wire ropes using unsaturated magnetic excitation and reconstruction image with super-resolution," Applied Sciences, vol. 8, no. 5, p. $767,2018$.
[19] L. Wie, Y. Ming, J. Luan, and Y. Guo, "Image fusion algorithm based on shift-invariant shearlet transform," Acta Photonica Sinica, vol. 42, no. 4, pp. 496-503, 2013.

[20] H.-Y. Cai, L.-R. Zhuo, P. Zhu, Z.-H. Huang, and X.-Y. Wu, "Fusion of infrared and visible images based on non-subsampled contourlet transform and intuitionistic fuzzy set," Acta Photonica Sinica, vol. 47, no. 6, 2018.

[21] L. N. Deng and X. F. Yao, "Research on the fusion algorithm of infrared and visible images based on non-subsampled shearlet transform," Acta Electronica Sinica, vol. 45, no. 12, pp. 2965-2970, 2017.

[22] G. Anbarjafari and H. Demirel, "Image super resolution based on interpolation of wavelet domain high frequency subbands and the spatial domain input image," ETRI Journal, vol. 32, no. 3, pp. 390-394, 2010.

[23] G. Gao, L. Xu, and D. Feng, "Multi-focus image fusion based on non-subsampled shearlet transform," IET Image Processing, vol. 7, no. 6, pp. 633-639, 2013.

[24] V. P. S. Naidu and J. R. Raol, "Pixel-level image fusion using wavelets and principal component analysis," Defence Science Journal, vol. 58, no. 3, pp. 338-352, 2008.

[25] P. Zhu, X. Ma, and Z. Huang, "Fusion of infrared-visible images using improved multi-scale top-hat transform and suitable fusion rules," Infrared Physics \& Technology, vol. 81, pp. 282-295, 2017.

[26] S. Aymaz and C. Köse, "A novel image decomposition-based hybrid technique with super-resolution method for multifocus image fusion," Information Fusion, vol. 45, pp. 113-127, 2019.

[27] J. Zhou, C. Zhou, J. Zhu, and D. Fan, “A method of superresolution reconstruction for remote sensing image based on non-subsampled contourlet transform," Acta Optica Sinica, vol. 35, no. 1, article 0110001, 2015.

[28] Y. Freund and R. E. Schapire, "A decision-theoretic generalization of on-line learning and an application to boosting," Journal of Computer and System Sciences, vol. 55, no. 1, pp. 119-139, 1997. 


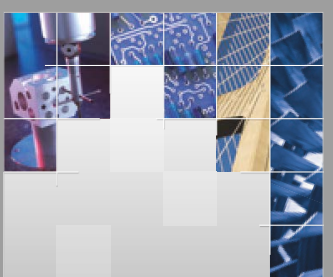

\section{Enfincering}
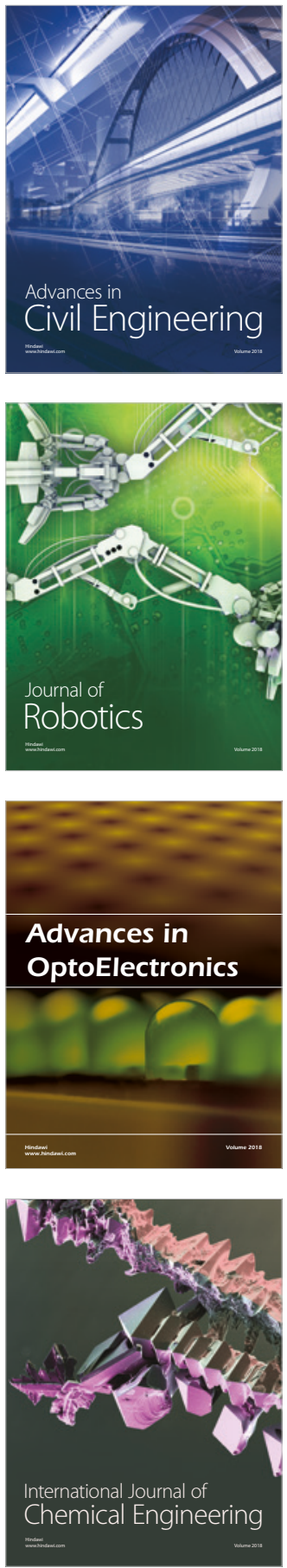

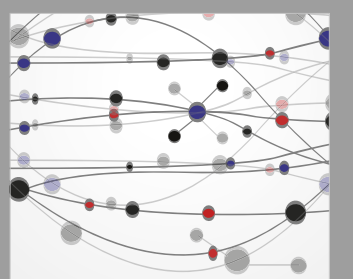

\section{Rotating \\ Machinery}

The Scientific World Journal

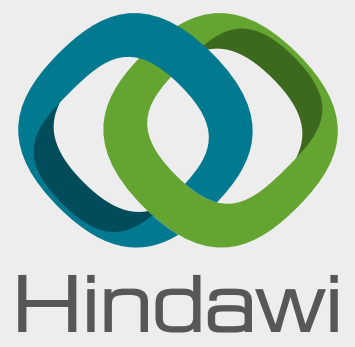

Submit your manuscripts at

www.hindawi.com
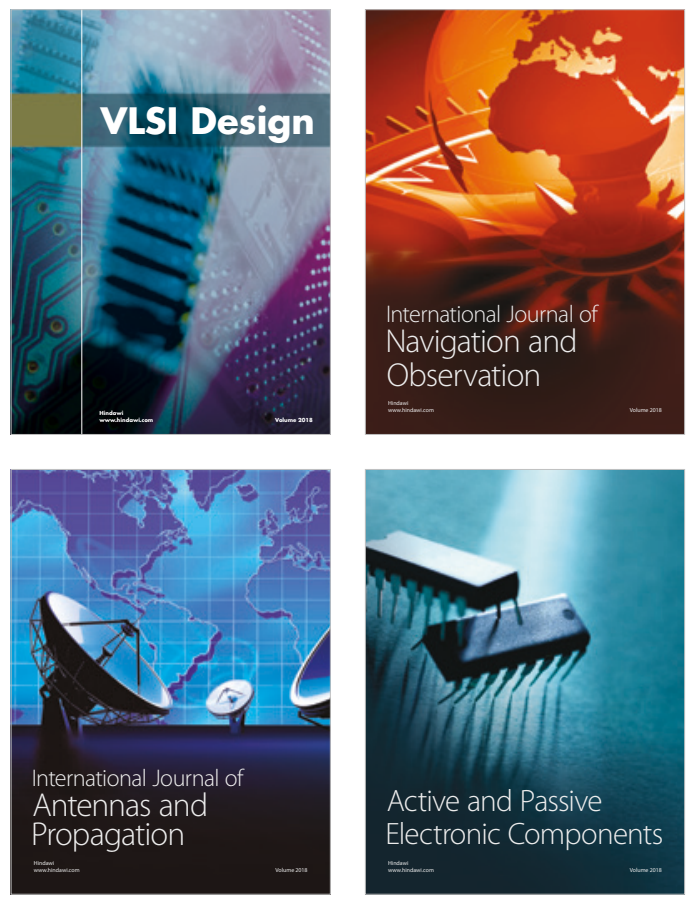
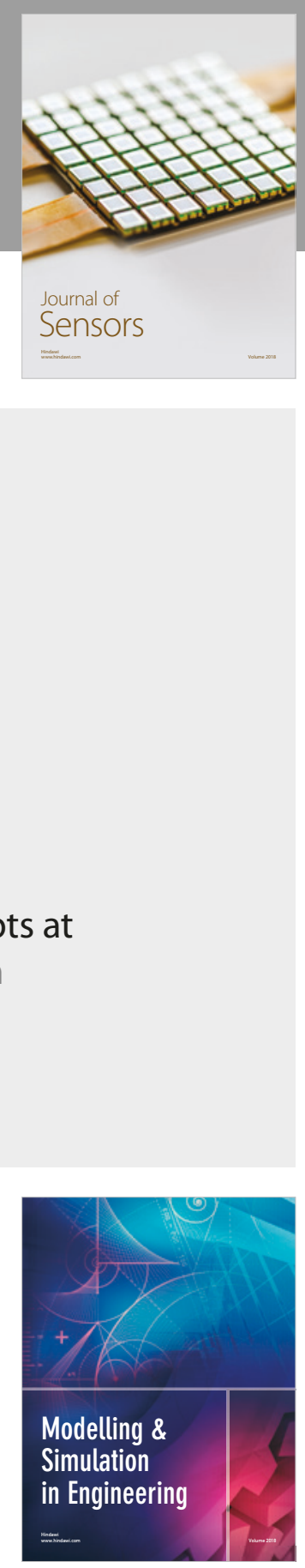

\section{Advances \\ Multimedia}
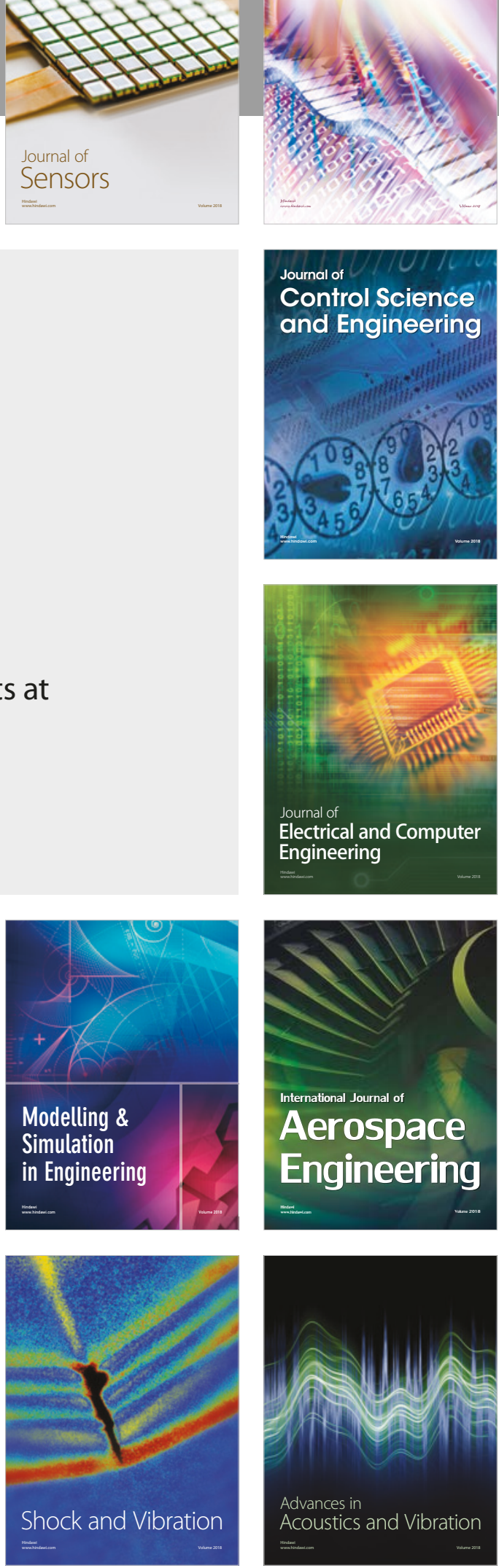